\title{
Lossless Deep Image Compression at the Edge for 3D Electron Microscopy
}

Jacob Hinkle $^{1}$, Todd Young ${ }^{2}$, Inzamam Haque ${ }^{3}$, Clay Reid ${ }^{4}$ and Olga Ovchinnikova ${ }^{5}$

${ }^{1}$ Oak Ridge National Laboratory, Oak Ridge, Tennessee, United States, ${ }^{2}$ Oak Ridge National Laboratory, United States, ${ }^{3}$ University of Tennnessee, United States, ${ }^{4}$ Allen Institute for Brain Science, United States, ${ }^{5}$ Computational Sciences and Engineering Division, Oak Ridge National Laboratory, Oak Ridge, Tennessee, United States

Recent progress in three-dimensional microscopy has enabled the collection of massive datasets with nanoscale resolution and millimeter-scale field of view. Advances like multibeam SEM promise to accelerate data acquisition leading to further increases in field of view, ultimately to a scale that entire mammalian brains can be imaged with sufficient resolution to trace individual neurons and synapses [1].

As data collection has advanced, so has our statistical modeling and computational capacity. In particular, our modern combined ability to acquire and model large datasets using deep learning (DL) has unleashed a new era in data-driven science, particularly in microscopy. It is now standard practice to incorporate DL into many phases of microscopy analysis pipelines.

While data acquisition and computation have taken great leaps forward, our computer networks and data storage facilities have advanced at a relatively slower pace. This presents a worrying situation in which we now worry that future advances in data acquisition will face insurmountable bottlenecks in transmission and storage. In this work, we explore the application of DL early in the analysis pipeline, for data reduction in order to facilitate faster and more economical transmission and storage from microscope to data center. This effort requires training deep neural networks before long-distance data transmission, using edge computing resources the like of which are currently being deployed at large-scale microscopy facilities.

Although deep learning has been applied recently to image compression (c.f. [2] and references therein), the field has entirely focused on the 2D natural images commonly found in commercial applications. Little, if any, attention has been paid to large three-dimensional datasets commonly found in medical and scientific applications. This has led to a difference in approach that values speed of (de)compression and generalization, problems that are relatively unimportant when dealing with much larger three-dimensional datasets. In our approach we train a network for each transmitted volumetric image block that uses a slicebased autoregressive context model to improve compression efficient, similar to that used in PixelCNN [3] but much more computationally efficient. The DL model we use is a simple convolutional neural network $(\mathrm{CNN})$ that predicts slices of a 3D volume using neighboring slices in one direction. This enables compression and decompression by marching through the volume in one direction, applying the CNN as a probabilistic context model that works in conjunction with standard entropy coding techniques.

We compared the DL approach to compression to PNG, a widespread compression technique that was developed at considerable expense. Our simple CNN architecture is able to predict slices accurately enough to outperform PNG with very little tweaking, as measured on a chunk of the Microns Layer 2/3 Phase 1 dataset [4,5] and shown visually in Fig. 1. Our results in Fig. 2 show that even lossless compression can benefit from DL, with a potential for much more improvement upon further refinement of our model architecture. 

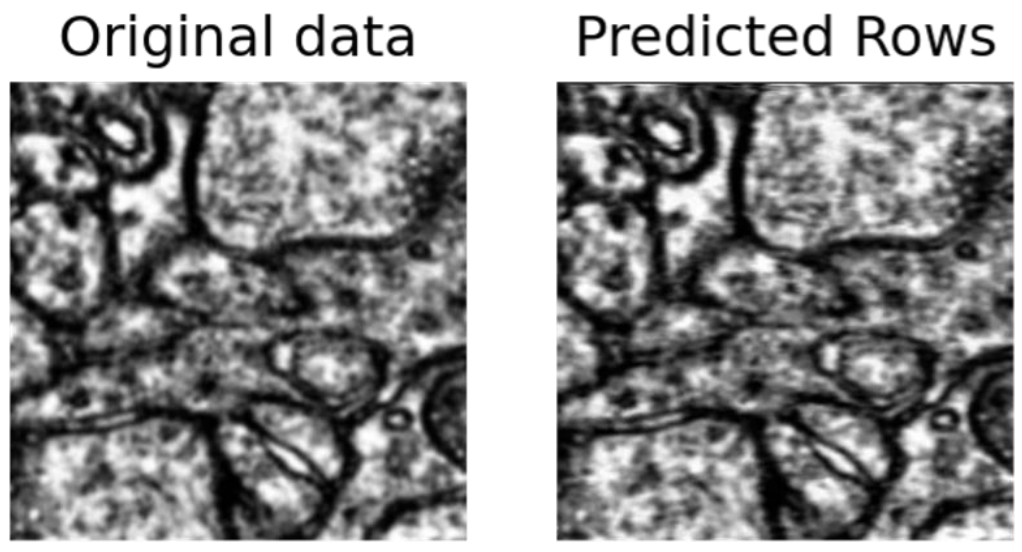

\section{Confidence}

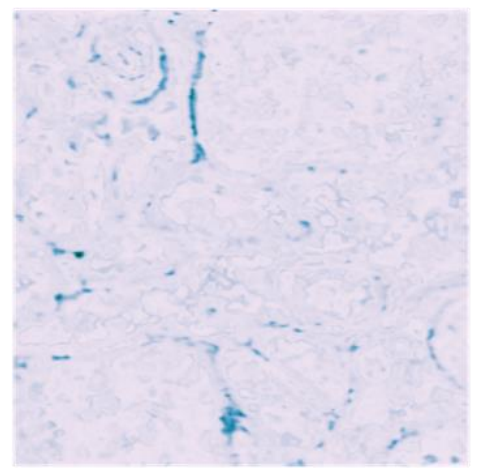

Figure 1. Sliced through 3D volume of EM data. (left) Original data, (center) each row predicted using deep context model with previous 4 observed slices, and (right) confidence in predicted rows with dark indicating increased confidence. These forward slice predictions match the original data well, leading to efficient compression when predictions match ground truth with high confidence. Note that the model is more confident in bold structures that align vertically, along the direction of prediction.

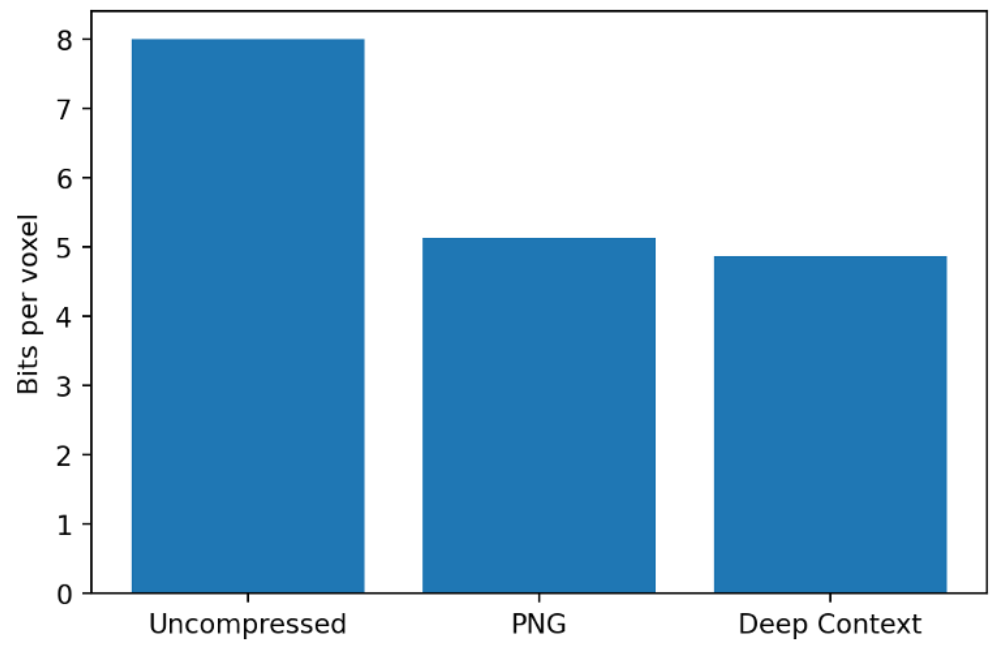

Figure 2. Bitrates for uncompressed 8-bit grayscale volume, PNG encoding, and deep learned context model. Our simple context model achieved bitrates surpassing the engineered PNG codec with very little customization.

\section{References}

[1] Abbott et al. Cell 182, 6 (2020).

[2] Mentzer et al. Proc. CVPR (2019).

[3] Oord et al. NIPS (2016).

[4] Dorkenwald et al. bioRxiv 2019.12.29.890319.

[5] Schneider-Mizell et al. bioRxiv 2020.03.31.018952v1. 